\title{
Plasma shape and finite $\beta$ effects on stability thresholds of the ion temperature gradient modes
}

\author{
B. Jhowry, J. Andersson, and S. Dastgeer \\ Department of Electromagnetics and Euratom/VR Association, Chalmers University of Technology, \\ S-41296 Göteborg, Sweden
}

(Received 5 February 2004; accepted 9 August 2004; published online 9 November 2004)

\begin{abstract}
The stability of electromagnetic ion temperature gradient driven modes with emphasis on the lower and upper stability thresholds is investigated by a collisionless magnetized plasma in both circular and noncircular geometry. The stability properties are discussed and the results are compared for finite $\beta$ effects, arbitrary elongation, and Shafranov shift rate parameters. It has been found that the lower stability thresholds are weakly dependent on the (combined) effects of Shafranov shift rate, finite $\beta$, and elongation whereas the second (upper) stability regime shows substantial dependence depending on parameter regimes. (C) 2004 American Institute of Physics.
\end{abstract} [DOI: 10.1063/1.1804941]

\section{INTRODUCTION}

Studies of confinement on shape and of magnetohydrodynamic (MHD) stability on toroidal plasma shape is well known and has been both dealt with theoretically and experimentally. However, theoretical microinstability growth rates in the high- $n$ linear ballooning mode formalism for the ion temperature gradient (ITG) modes with emphasis on plasma shape should be given full attention. Elongation effects have shown decrease in growth rates in the plasma core for ITG modes as given in Ref. 1. Studies of trapped electron drift modes showed that increasing the elongation leads to a decrease in growth rate for these modes and for the ITG mode the growth rate decreases as elongation increases in the core region see, for example, Refs. 2 and 3. Collisional ITG mode in cross-sectional elongation and Shafranov shift on the stability of resistive edge modes have been investigated as given in Ref. 4. In circular geometry it was shown that the ion temperature gradient mode stabilizes for high values of $\left(\eta_{i}=L_{n} / L_{T i}\right.$, where $L_{n}=n|d n / d r|^{-1}$ and $L_{T i}=T_{i}\left|d T_{i} / d r\right|^{-1}$ are the characteristic length scales of the density and ion temperature), see, for example, Ref. 5. Studies in both fluid and gyrokinetic models for the upper stability ( $\eta_{\text {iup }}$ threshold with finite $\beta$ (=plasma pressure/magnetic pressure) effects have shown stabilization at high $\eta_{i}$ values as given in Ref. 6.

Plasma shape parameters such as elongation $\kappa(=b / a)$ (elliptic surface/circular surface) and Shafranov shift rate $\left(\Delta=\partial_{r} R_{0}\right)$ see, for example, Ref. 7 in toroidal devices play an important role in the power scaling expressions for thermal energy confinement time, $\tau_{t h}$, which can be expressed either in "engineering" variables as $\tau_{\text {th }}$ $=C I^{\alpha_{I}} B^{\alpha_{B}} P^{\alpha_{P}} n^{\alpha_{n}} M^{\alpha_{M}} R^{\alpha_{R}} \varepsilon^{\alpha_{\varepsilon}} \kappa^{\alpha_{\kappa}}$ and in "physics" variables as $\tau_{t h}=C_{1} \tau_{B}^{x_{r_{B}}} \rho_{*}^{x_{*} \rho^{*}} \nu_{*}^{x_{*}} \beta^{x_{\beta}} M^{x_{M}} q^{x_{q}} \varepsilon^{x_{r}} \kappa^{x_{\kappa}}$ where $R$ is major radius, $I$ the plasma current, $B$ the toroidal magnetic field (at major radius $R$ ), $P$ the power loss (corrected for charge exchange and orbit losses), $n$ the line average density, $\kappa$ the elongation, $\epsilon$ the inverse aspect ratio and $M$ the average ion mass. The "physics" variables are $\tau_{B}$ the Bohm time, the normalized variables are the toroidal Larmor radius $\rho_{*}$, the collisionality $\nu_{*}$, the plasma pressure $\beta, \Delta$ appears indirectly through $\beta$, the cylinder safety factor $q$.

Plasma shaping, finite- $\beta$ stabilization, and Shafranov shift may affect the formation and sustainment of confinement regimes by the creation of a heat transport barrier. $\eta_{i}$ should be large enough that the growth rate should decrease with increasing $\eta_{i}$. Transport barriers with large $\eta_{i}$ have been observed in all major tokamaks, see, for example, Ref. 8. It has been reported that effects such as sheared rotation, finite $\beta$, and plasma shaping be considered in reducing the growth rates see, for example, Ref. 9.

In this paper we investigate the elongation $\kappa=b / a$, electromagnetic (finite $\beta$ ) and Shafranov shift $\Delta\left(=\partial_{r} R_{0}\right)$, see, for example, Ref. 7 effects on the lower $\eta_{\text {ilow }}$ and upper $\eta_{\text {iup }}$ stability thresholds of the ITG modes. We are specially interested in the effects (combined) of finite $\beta$, elongation parameter $\kappa$, and Shafranov shift $\Delta$ on the ITG mode particular to noncircular geometry and with various key plasma parameters. We compare the results with the one obtained in circular geometry in the first and second stability thresholds (i.e., fluid models give two marginal stability limits as given in Ref. 10).

Here we have modified the advanced fluid model see, for example, Refs. 11 and 12 and stability code to treat equilibria with an arbitrary elongation $\kappa$ of the cross section and with arbitrary Shafranov shift rate $\Delta$. The work is focused on the collisionless ITG ballooning modes in low $\beta$ tokamak equilibrium ( $s$ - $\alpha$ model). The frequency regime of interest is $\omega_{T_{i}}<|\omega| \ll \omega_{T_{e}}$ where $\omega_{T}$ is the transit frequency. The model equations investigated here contain the MHD ballooning mode generalized to finite $k_{\perp}^{2} \rho_{i}^{2}$ finite Larmor radius (FLR), and general $\omega_{D} / \omega$ see, for example, Refs. 13 and 12. In the MHD expansion the stability is determined by the parameters $\alpha=\beta q^{2} R / L_{n}\left[1+\eta_{e}+\left(1+\eta_{i}\right) / \tau\right] \tau /(\tau+1)$ and $s=r q^{\prime} / q$ with $\tau=T_{e} / T_{i}$. Parallel ion dynamics and trapped particle effects are ignored.

The sensitivity of drift waves to plasma shape depends strongly on the fluid closure. Hence to clarify the effects of elongation with an advanced fluid model is essential. The 
modified advanced fluid model has successfully reproduced experimental and nonlinear gyrokinetic results, see, for example, Ref. 14. Furthermore, this advanced fluid model has shown to exhibit an excellent agreement with the full 3D nonlinear gyrokinetic particle simulation in the estimation of ion heat diffusivity coefficient, under the Cyclone project, see, for example, Ref. 15.

The remainder of the paper is organized as follows: In Sec. II, the model is presented and the eigenmode equation is derived. In Sec. III the simulation results are discussed and in Sec. IV a summary is given.

\section{MODEL FORMULATION}

The fluid model used here is the ion fluid model with Boltzmann electrons. The model equations are solved by utilizing a generalized noncircular equilibrium mode ${ }^{16}$ which allows for modification of arbitrary elongation and Shafranov shift. The flux coordinate system is given by

$$
\begin{aligned}
& R(r, \theta)=\sum_{n=0}^{\infty} R_{n} \cos (n \theta), \\
& Z(r, \theta)=\sum_{n=0}^{\infty} Z_{n} \sin (n \theta) .
\end{aligned}
$$

Here $R$ and $Z$ are the usual cylinder coordinates. The terms $R_{0}, R_{1}=r, Z_{1}=r \kappa$ describes shift, minor radius and ellipticity, respectively. In Eqs. (1) and (2) $R_{2}, Z_{2}$ describes triangularity which is neglected in this work. The Riemann metric tensor $\widetilde{g}_{i j}$ Ref. 17 and 18 is usually used in modeling the magnetic flux surfaces and is defined as

$$
d s^{2}=\widetilde{g}_{r r} d r^{2}+\tilde{g}_{\theta \theta} d \theta^{2}+2 \tilde{g}_{r \theta} d r d \theta+\tilde{g}_{\phi \phi} d \phi^{2}=\tilde{g}_{i j} d x^{i} d x^{j},
$$

where $\widetilde{g}_{r r}, \widetilde{g}_{\theta \theta}, \widetilde{g}_{r \theta}, \widetilde{g}_{\phi \phi}$ are the relevant normalized (by $R$ and $r$ ) elements of the metric tensor $\tilde{g}_{i j} \tilde{g}^{j k}=\delta_{i}^{k}$ defining the geometry. The inverse of the metric tensor is deduced from the relation $\widetilde{g}_{i j} \widetilde{g}^{i k}=\delta_{i}^{k}$ where $\delta_{i}^{k}$ is the Kronecker $\delta$ :

$$
\begin{aligned}
& \widetilde{g}^{r r}=\frac{\widetilde{g}_{\theta \theta}}{\widetilde{g}_{r r} \widetilde{g}_{\theta \theta}-\widetilde{g}_{r \theta}^{2}}, \\
& \widetilde{g}^{\theta \theta}=\frac{\widetilde{g}_{r r}}{\widetilde{g}_{r r} \widetilde{g}_{\theta \theta}-\widetilde{g}_{r \theta}^{2}}, \\
& \widetilde{g}^{r \theta}=\widetilde{g}_{\theta r}=-\frac{\widetilde{g}_{r \theta}}{\widetilde{g}_{r r} \widetilde{g}_{\theta \theta}-\widetilde{g}_{r \theta}^{2}}, \\
& \partial_{r} R_{0}=\Delta, \\
& \widetilde{g}_{r r}=(\Delta+\cos \theta)^{2}+(\kappa \sin \theta)^{2}, \\
& \widetilde{g}_{\theta \theta}=1+\left(\kappa^{2}-1\right) \cos \theta^{2}, \\
& \widetilde{g}_{r \theta}=\widetilde{g}_{\theta r}=\sin \theta\left[\kappa^{2} \cos \theta-(\Delta+\cos \theta)\right], \\
& \widetilde{g}_{\phi \phi}=1,
\end{aligned}
$$

$$
\begin{aligned}
& \widetilde{g}_{\phi r}=\widetilde{g}_{r \phi}=\widetilde{g}_{\phi \theta}=\widetilde{g}_{\theta \phi}=0, \\
& \widetilde{J}^{2}(r, \theta)=\widetilde{g}_{\phi \phi}\left(\widetilde{g}_{r r} \widetilde{g}_{\theta \theta}-\widetilde{g}_{r \theta}^{2}\right) .
\end{aligned}
$$

Here $\kappa$ is the elongation, and Eq. (13) is the normalized Jacobian. The elements of the inverse metric tensor $\tilde{g}^{i j}$ are given by

$$
\begin{aligned}
& \widetilde{g}^{r r}=\widetilde{g}_{\theta \theta} \widetilde{g}_{\phi \phi} \widetilde{J}^{2}(r, \theta), \\
& \widetilde{g}^{\theta \theta}=\widetilde{g}_{r r} \widetilde{g}_{\phi \phi} \widetilde{J}^{2}(r, \theta), \\
& \widetilde{g}^{r \theta}=\widetilde{g}^{\theta r}=-\widetilde{g}_{r \theta} \widetilde{g}_{\phi \phi} \widetilde{J}^{2}(r, \theta), \\
& \widetilde{g}^{\phi \phi}=1, \\
& \widetilde{g}^{\phi r}=\widetilde{g}^{r \phi}=\widetilde{g}^{\phi \theta}=\widetilde{g}^{\theta \phi}=0,
\end{aligned}
$$

and the Christoffel symbols $\widetilde{\Gamma}_{j k}^{i}$ are

$$
\begin{aligned}
& \widetilde{\Gamma}_{\phi \phi}^{r}=\widetilde{g}^{r r} \sin \theta-\widetilde{g}^{r r}(\Delta+\cos \theta), \\
& \widetilde{\Gamma}_{\phi \phi}^{\theta}=\widetilde{g}^{\theta \theta} \sin \theta-\widetilde{g}^{r \theta}(\cos \theta+\Delta) .
\end{aligned}
$$

In deriving the normalized Eqs. (19) and (20) we have used the non-normalized expressions

$$
\begin{aligned}
& g_{\phi \phi}=R^{2}, \\
& g^{r r}=\frac{g_{\theta \theta}}{g_{r r} g_{\theta \theta}-g_{r \theta}^{2}}, \\
& g^{\theta \theta}=\frac{g_{r r}}{g_{r r} g_{\theta \theta}-g_{r \theta}^{2}}, \\
& g^{r \theta}=g^{\theta r}=\frac{g_{r \theta}}{g_{r r} g_{\theta \theta}-g_{r \theta}^{2}}, \\
& \Gamma_{\phi \phi}^{r}=-\frac{1}{2}\left(g^{\theta \theta} \partial_{r} g_{\phi \phi}+g^{r \theta} \partial_{\theta g_{\phi \phi}}\right), \\
& \Gamma_{\phi \phi}^{\theta}=-\frac{1}{2}\left(g^{\theta \theta} \partial_{\theta g_{\phi \phi}}+g^{r \theta} \partial_{r} g_{\phi \phi}\right) .
\end{aligned}
$$

By introducing $\mathbf{B}=B_{\theta} \hat{\theta}+B_{\phi} \hat{\phi}$ (with $\theta$ and $\phi$ the poloidal and toroidal directions respectively), $e_{\|}=\mathbf{B} / B, \boldsymbol{\nabla}=\boldsymbol{\nabla} r \partial_{r}$ $+\nabla \theta \partial_{\theta}+\nabla \phi \partial_{\phi}$, we obtain the parallel structure operator as

$$
k_{\|}=e_{\|} \cdot \nabla=\frac{B_{\phi}}{B h_{\phi}} \partial_{\phi}+\frac{B_{\theta}}{B h_{\theta}} \partial_{\theta},
$$

where $h_{\theta}=\sqrt{\widetilde{g}_{\theta \theta}}$ and $h_{\phi}=\sqrt{\widetilde{g}_{\phi \phi}}=1$. We note that there is no $\phi$-dependence (toroidal axis symmetry) on $g_{\theta \theta}, g_{\phi \phi}, B_{\theta}$, and $B_{\phi}$. This leads to

$$
\begin{aligned}
\left(e_{\|} \cdot \nabla\right) e_{\|}= & \frac{1}{B^{2}}\left(\frac{B_{\phi}^{2}}{h_{\phi}^{2}} e_{j}+\frac{B_{\theta} B_{\phi}}{h_{\theta}}\left(\partial_{\theta} h_{\theta}^{-1} e_{\theta}+h_{\theta}^{-1} \Gamma_{\theta \theta}^{j} e_{j}\right.\right. \\
& \left.+\partial_{\theta} h_{\phi}^{-1} e_{\phi}+\left[h_{\theta}^{-1}+h_{\phi}^{-1}\right] \Gamma_{\theta \theta}^{j} e_{j}\right)+\frac{B_{\theta}}{h_{\theta}^{2}} \partial_{\theta} B_{\theta} e_{\theta} \\
& \left.+\frac{B_{\theta}}{h_{\theta} h_{\phi}} \partial_{\theta} B_{\phi} e_{\phi}\right)+\| \text { terms. }
\end{aligned}
$$


Here $\Gamma_{\phi \theta}^{j}=\Gamma_{\theta \phi}^{j}=0,(j=r, \theta)$ where $\Gamma_{\theta \theta}^{j}, \Gamma_{\phi \theta}^{j}$, and $\Gamma_{\theta \phi}^{j}$ are nonnormalized quantities. The terms parallel to $B$ have been neglected because they will be canceled when the cross product $e_{\|} \times\left(e_{\|} \cdot \nabla\right) e_{\|}$(which is $\left.\mathbf{v}_{D}\right) . k_{\perp}$ is calculated using the WKB aproximation, i.e.,

$$
\nabla_{\perp} f \approx i k_{\perp} f, f=\tilde{f} e^{-i\left(\int \nu d \theta \pm \phi\right)}
$$

is the eikonal assumption with $\nu=\nu(r, \theta)$. By using, Eq. (3) we now obtain $\mathbf{k}_{\perp}$ as $n\left(\nabla r \partial_{r} q+\nabla \theta \partial_{\theta} q-\nabla \phi\right)$. Through some tedious algebra $\mathbf{k}_{\perp} \cdot e_{\|} \times\left(e_{\|} \cdot \boldsymbol{\nabla}\right) e_{\|}$can be calculated. Finally we obtain the factor $\widetilde{g}(\theta)$ (poloidal variation of the magnetic field) through space variation $\mathbf{k}_{\perp} \cdot \mathbf{v}_{D}=\omega_{D} \widetilde{g}(\theta)$ as

$$
\begin{aligned}
\widetilde{g}(\theta)= & \frac{1}{\sqrt{\widetilde{g}_{\phi \phi}}} \widetilde{J}^{2}(r, \theta) q s\left[\widetilde{\Gamma}_{\phi \phi}^{r}\left(\widetilde{g}^{r \theta} s \theta+\widetilde{g}^{\theta \theta}\right)\right. \\
& \left.-\widetilde{\Gamma}_{\phi \phi}^{\theta}\left(\widetilde{g}^{r \theta}+\widetilde{g}^{r r} s \theta\right)\right] .
\end{aligned}
$$

$\mathbf{k}_{\perp}$ is the propagation vector perpendicular to the magnetic field $\mathbf{B}$ and originates from Eq. (3) (Refs. 17 and 18). For small $k_{\|}$the motion of the electrons along the field lines is less efficient for space charge cancellations. This is the reason for our interest in modes with $k_{\perp} \gg k_{\|}$, which is the main variation of the mode in the perpendicular plane. Here $s$ $=r q^{\prime} / q$ and $q$ is the safety factor. Here $q$ and $s$ appear in $\mathbf{k}_{\perp}=n\left(\nabla r \partial_{r} q+\nabla \theta \partial_{\theta} q-\nabla \phi\right)$ and $\mathbf{v}_{D}$ (sum of $\boldsymbol{\nabla} B$ and curvature drifts).

The magnetic drift frequency $\omega_{D}$ and $k_{\perp}$ are given by

$$
\begin{aligned}
& k_{\perp}^{2}=k_{\theta}^{2} k(\theta) \approx-\frac{k_{\theta}^{2}}{\widetilde{J}^{2}(r, \theta)}\left[\widetilde{g}_{r r}+\widetilde{g}_{\theta \theta}(s \theta)^{2}+2 \widetilde{g}_{r \theta} s \theta\right], \\
& k(\theta)=-\frac{1}{\widetilde{J}^{2}(r, \theta)}\left[\widetilde{g}_{r r}+\widetilde{g}_{\theta \theta}(s \theta)^{2}+2 \widetilde{g}_{r \theta} s \theta\right],
\end{aligned}
$$

where

$$
\begin{aligned}
& \mathbf{k}_{\perp} \cdot \mathbf{v}_{D}=\omega_{D} \tilde{g}(\theta), \\
& \omega_{D}=\varepsilon_{n} \omega_{*_{e}}, \\
& \varepsilon_{n}=2 L_{n} / L_{B} .
\end{aligned}
$$

$\omega_{*_{e}}$ is the electron diamagnetic frequency.

For $\Delta=0$ and $\kappa=1$ the magnetic drift in circular geometry can be deduced from the above results with $\widetilde{g}(\theta)$ $=\cos \theta+s \theta \sin \theta$ and $k(\theta)=1+s^{2} \theta^{2}$. A low $\beta$ equilibrium (tokamak discharge) with elongation $(\kappa)$ effects is assumed. The perturbed electric field is represented by the scalar potential $\phi$ and the parallel vector potential $A_{\|}$. The compressional Alfvén (magnetosonic) mode is omitted. The field equations are the parallel Ampères law

$$
\nabla_{\perp}^{2} A_{\|}=\frac{4 \pi}{c} J_{\|},
$$

and the quasineutrality condition

$$
\delta n_{e}=\delta n_{i},
$$

where $c$ is the speed of light, $J_{\|}$is the current parallel to the magnetic field, and $\delta n_{i(e)}$ is the electron (ion) density pertur- bation. We have assumed $|\omega| \sim k_{\|} v_{A} \ll k_{\|} v_{t e}$ in the regime under consideration and the parallel current perturbation is primarily carried by the free electrons. The current and electron density perturbations are derived from the parallel electron momentum and continuity equations leading to (see, for example, Refs. 12, 13, and 19 for details):

$$
\begin{aligned}
J_{\|}= & J_{\|_{e}}=\frac{n_{0} e^{2}}{k_{\|} T_{e}}\left(\left(\omega_{*_{e}}-\omega\right) \phi\right. \\
& \left.+\frac{\left(\omega-\omega_{*_{e}}\right)\left(\omega-\omega_{D e}\right)+\eta_{e} \omega_{*_{e}} \omega_{D e}}{c k_{\|}} A_{\|}\right)
\end{aligned}
$$

and

$$
\frac{\delta n_{e}}{n_{0}}=\left(\phi-\frac{\omega-\omega_{* e}}{c k_{\|}} A_{\|}\right) \frac{e}{T_{e}},
$$

where $e$ is the electron charge, $T_{e}$ the electron temperature, $n_{0}$ the background density, $\omega$ the eigenfrequency, and $\omega_{D e}$ is the magnetic drift frequency, and $\eta_{e}=d\left[\ln \left(T_{e}\right)\right] / d[\ln (n)]$ $=L_{n} / L_{T_{e}}$. The two-fluid ion model includes first order FLR terms, the polarization drift and the compressibility effects due to the field curvature (in particular, the contribution from the divergence of the diamagnetic heat flow in the energy equation). The ion density response is deduced by combining the energy equation and the ion continuity equation leading to (see, for example, Refs. 12 and 19)

$$
\frac{\delta n_{i}}{n_{0}}=Q(k, \omega, \theta)\left(\frac{e \phi}{T_{i}}\right)
$$

where

$$
Q(k, \omega, \theta)=\frac{T}{N},
$$

and

$$
\begin{aligned}
T= & \omega\left(\omega_{D i}-\omega_{*_{i}}\right)+\left(\frac{7}{3}-\eta_{i}\right) \omega_{D i} \omega_{*_{i}}-\frac{5}{3} \omega_{D i}^{2}-b\left(\omega-\omega_{*_{i P}}\right) \\
& \times\left(\omega-\frac{5}{3} \omega_{D i}\right), \\
N= & \omega^{2}-\frac{10}{3} \omega \omega_{D i}+\frac{5}{3} \omega_{D i}^{2},
\end{aligned}
$$

where $\omega_{*_{i}}$ is the ion diamagnetic drift frequency $\left(\omega_{*_{e}}=-\tau \omega_{*_{i}}\right), \omega_{D i}$ is the ion magnetic drift (curvature and $\nabla \mathbf{B}$ drifts) frequency, $\quad \omega_{D e}=-\tau \omega_{D i}, \quad \omega_{*_{i P}}=\omega_{*_{i}}\left(1+\eta_{i}\right), \quad \eta_{i}$ $=d\left[\ln \left(T_{i}\right)\right] / d[\ln (n)]=L_{n} / L_{T i}, L_{n}$ is the density scale length $\left(L_{j}=\left|(d[\ln (j)] / d r)^{-1}\right|\right), \quad \tau=T_{e} / T_{i}, \quad b=\left(k_{\perp} \rho\right)^{2}$, and $\tau \rho^{2} / 2=\rho_{s}^{2}$ $=\left(c_{s} / \omega_{c i}\right)^{2}$, where $c_{s}^{2}=T_{e} / m_{i}$ and $\omega_{c i}=e B / m_{i} c$. We note that the ion density perturbation is purely electrostatic. Using the standard high $n$ ballooning mode formalism as given in Ref. 20, the parallel Ampères law and the quasineutrality condition (17) leads to the following eigenvalue differential equation for the reduced potential $\phi=A_{\|} / k_{\|}$:

$$
\frac{1}{h_{\theta}} \frac{d}{d \theta}\left(\frac{k_{\perp}^{2}(\theta)}{k_{\theta}^{2}}\right) \frac{1}{h_{\theta}} \frac{d \phi}{d \theta}+\beta \frac{2}{1+1 / \tau}\left(\frac{q}{\varepsilon_{n}}\right)^{2} V(k, \bar{\omega}, \theta) \phi=0,
$$

$$
h_{\theta}=\sqrt{g_{\phi \phi}},
$$




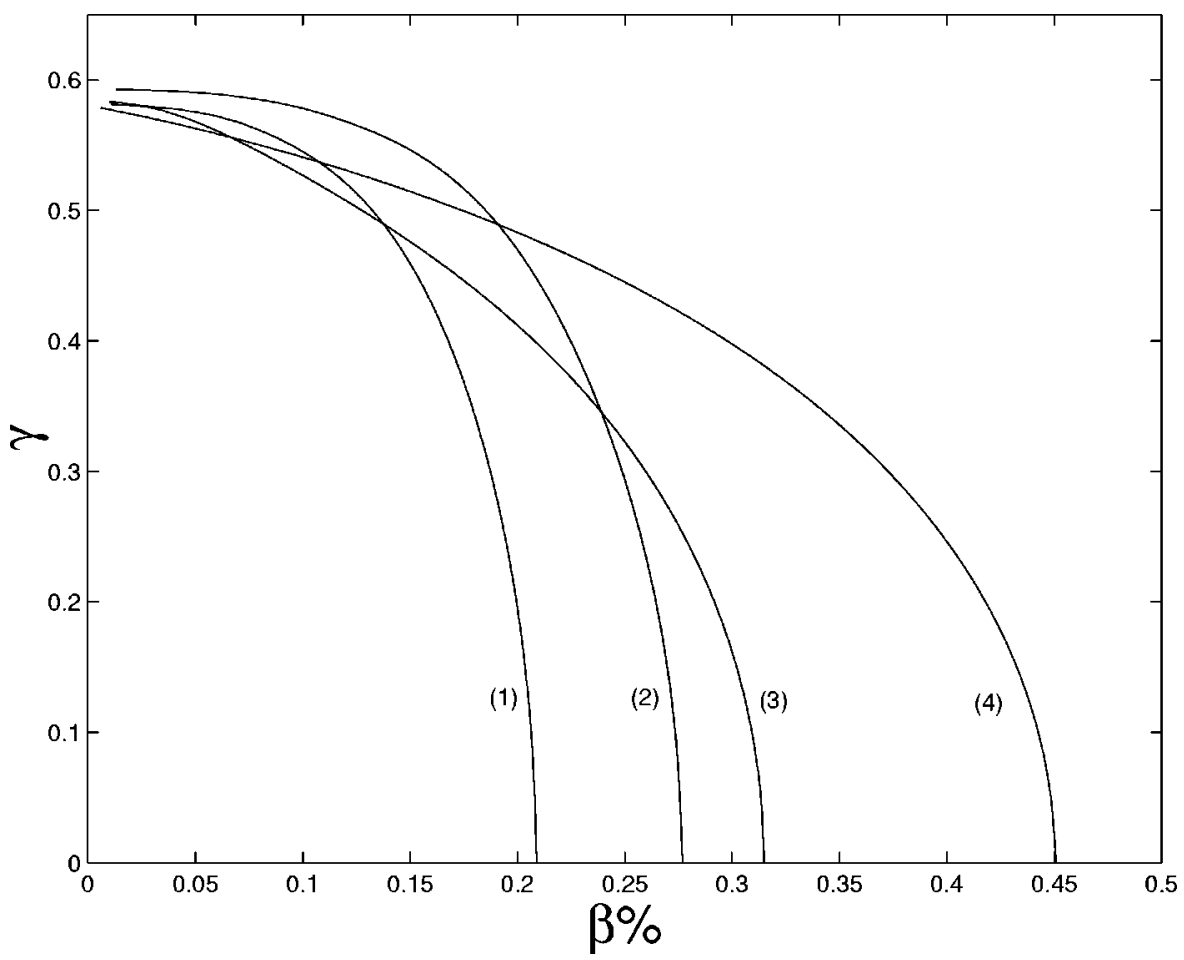

FIG. 1. The growth rate $\gamma$ (normalized by $\left.\omega_{*}\right)$ vs $\beta$ with varying parameters $\Delta(=-0.0,-0.1)$ and $\kappa(=1,1.5)$. The curves (1) and (2) have shift $\Delta=-0.1$ and $\Delta=0$, respectively, with fixed elongation $\kappa=1.5$. The curves (3) and (4) have shift $\Delta=-0.1$ and $\Delta=0$, respectively, with fixed $\kappa=1$. Other parameters are $\varepsilon_{n}=0.5, \eta=2, q=2, k^{2} \rho^{2}$ $=0.1, s=0.5$, and $\tau=1$.

$$
\beta=\frac{8 \pi n\left(T_{e}+T_{i}\right)}{B^{2}},
$$

and $k_{\perp}^{2}(\theta)$ is as in Eq. (15). Here $\beta$ is the plasma beta, $q$ is the safety factor, $\theta$ is the extended poloidal angle, and $\alpha$ is a ballooning parameter. The potential function $V$ is given by

$$
\begin{aligned}
V(k, \bar{\omega}, \theta)= & (\bar{\omega}-1)\left[\bar{\omega}-\varepsilon_{n} g(\theta)\right]+\eta_{e} \varepsilon_{n} g(\theta) \\
& -\frac{(\bar{\omega}-1)^{2}}{1-\tau Q(k, \bar{\omega}, \theta)}
\end{aligned}
$$

Here $\bar{\omega}$ is the normalized eigenfrequency $\left(\omega / \omega_{* e}\right), \widetilde{g}(\theta)$

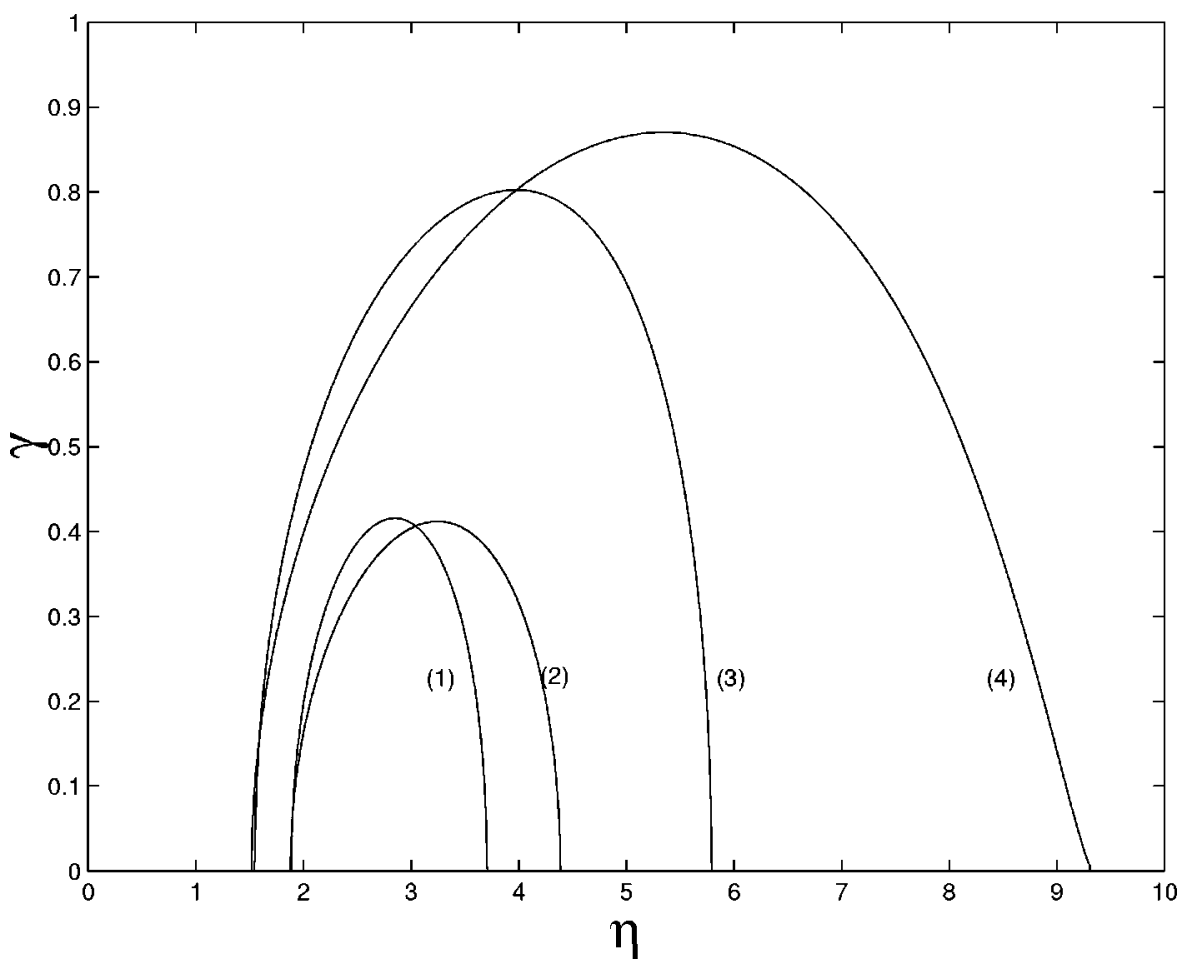

FIG. 2. The growth rate $\gamma$ (normalized by $\left.\omega_{*_{e}}\right)$ as a function of $\eta_{i e}=\eta_{i}=\eta_{e}$ with fixed parameters $\Delta(=-0.0,-0.1)$ and $\kappa(=1,0,1.5)$. Other parameters are $k^{2} \rho^{2}=0.1, q=2, s=0.5, \varepsilon_{n}=0.5, \beta$ $=0.3 \%$, and $\tau=1$. The curves (1) and (2) have elongation $\kappa=1.5$ and $\kappa=1.0$, respectively, with fixed Shafranov shift $\Delta=-0.1$. The curves (3) and (4) have elongation $\kappa=1.5$ and $\kappa=1.0$, respectively, and fixed $\Delta=-0.0$. The curves (1) and (3) have $\beta=0.2 \%$ and curves (2) and (4) have $\beta=0.3 \%$. 


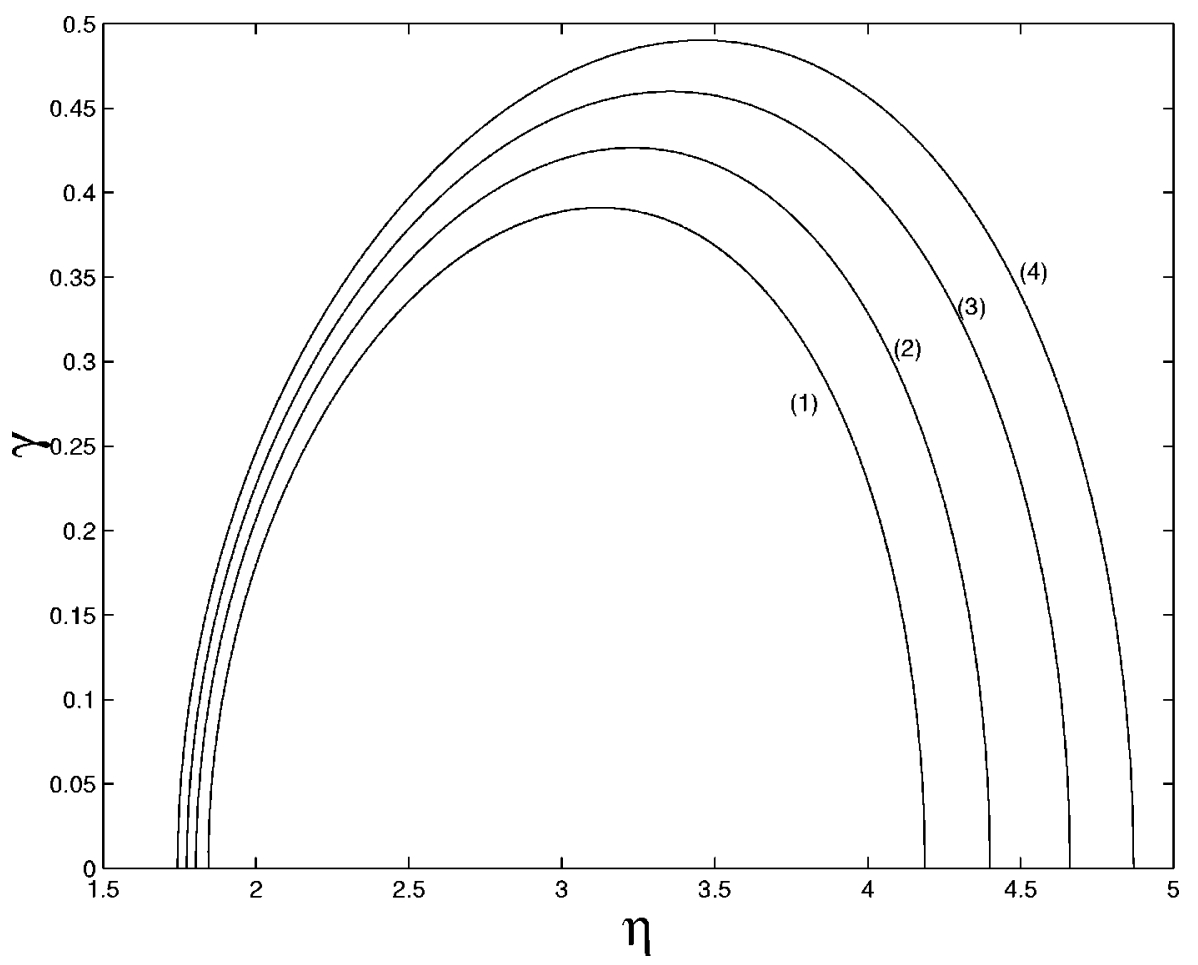

FIG. 3. The growth rate $\gamma$ (normalized by $\left.\omega_{* e}\right)$ as a function of $\eta_{i e}=\eta_{i}=\eta_{e}$ with fixed parameters $\Delta(=-0.0$, $-0.005)$ and $\kappa(=1,1.05)$. Other parameters are $k^{2} \rho^{2}=0.1, q=2, s=0.5, \varepsilon_{n}$ $=0.5, \beta=0.4 \%$ and $\tau=1$. The curves (1) and (2) have Shafranov shift $\Delta=-0.005$ and $\Delta=-0.0$, respectively, with fixed elongation $\kappa=1.05$. The curves (3) and (4) have Shafranov shift $\Delta=-0.005$ and $\Delta=-0.0$, respectively, with fixed elongation $\kappa=1.0$. The curve (5) has $\kappa=1.0$ and $\Delta=-0.0$ and with $\beta=0.1 \%$.

given by Eq. (30) above represents the normalized poloidal variation of $\omega_{D}$ in noncircular geometry, $s$ $=d[\ln (q)] / d[\ln (r)]$ is the shear parameter, and $Q$ is given by Eq. (41). The derived eigenvalue differential equation (44) describes the finite $\beta$ modified $\eta_{i}$ mode in the toroidal limit and the electromagnetic ballooning mode. The boundary conditions for the potential $\phi$ and the extended poloidal angle in toroidal geometry $\theta$ are given by

$$
\begin{aligned}
& \frac{d \phi}{d \theta}(\theta=0)=0 \\
& \phi(\theta \rightarrow \infty) \rightarrow 0 .
\end{aligned}
$$

The eigenvalues $\bar{\omega}$ are iterated until the boundary condition $\phi(\theta \rightarrow \infty) \rightarrow 0$ (Ref. 20) is satisfied. In this work we have assumed $T_{e}=T_{i}, \eta=\eta_{i}=\eta_{e}$. The plasma shape parameters

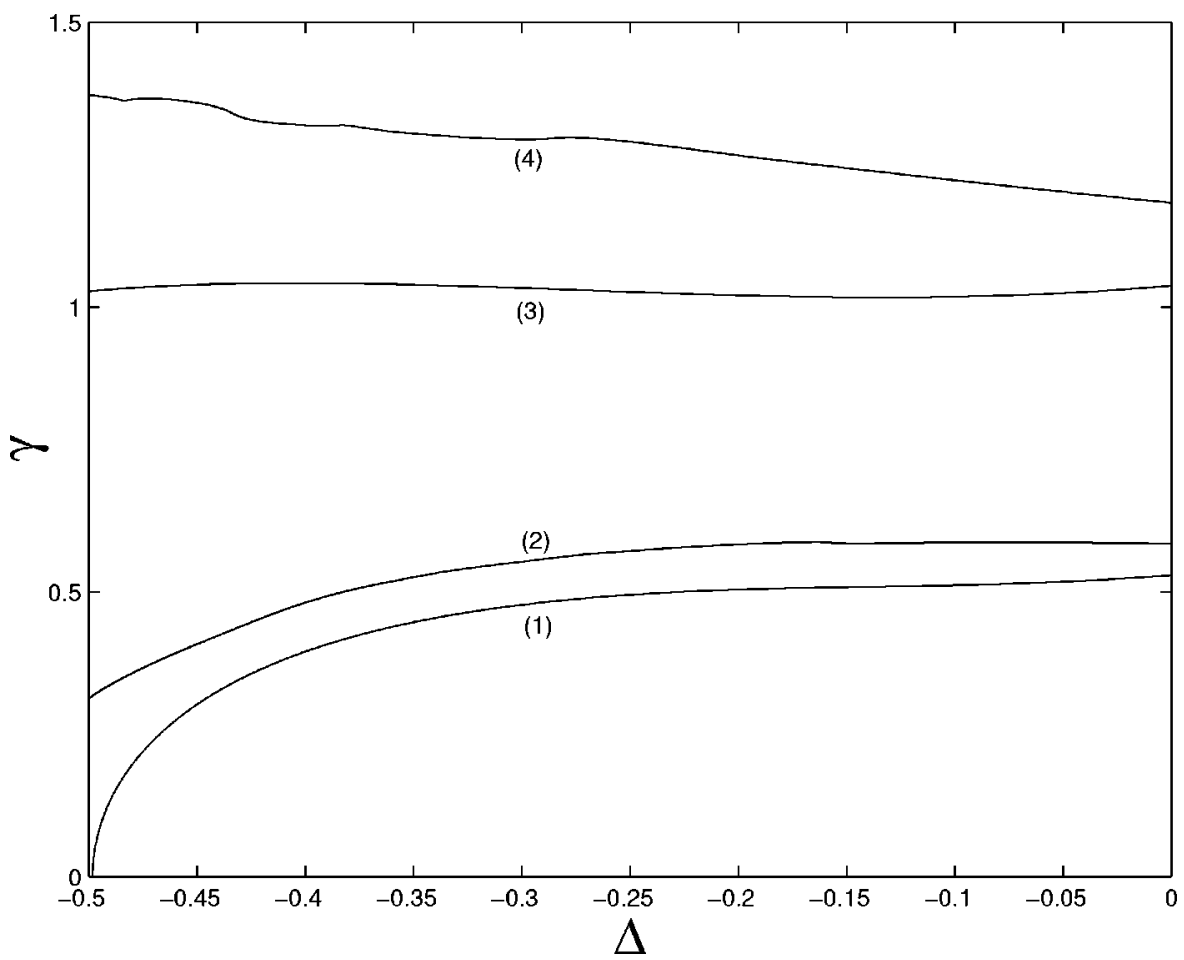

FIG. 4. The dependence of growth rate $\gamma$ (normalized by $\omega_{*}$ ) as a function of $\Delta$ with $\kappa=(1,1.5)$ and $\eta$ $=(2,4)$ as parameters. Curves $(1)$ and (2) have $\kappa=1$ and $\kappa=1.5$, respectively, with $\eta=2$ ). Curves (3) and (4) have $\kappa=1$ and $\kappa=1.5$, respectively, with $\eta$ $=4)$. The other parameters are $\beta$ $=0.001, \varepsilon_{n}=0.5, q=2, k^{2} \rho^{2}=0.1, \tau=1$, $s=0.5$. 


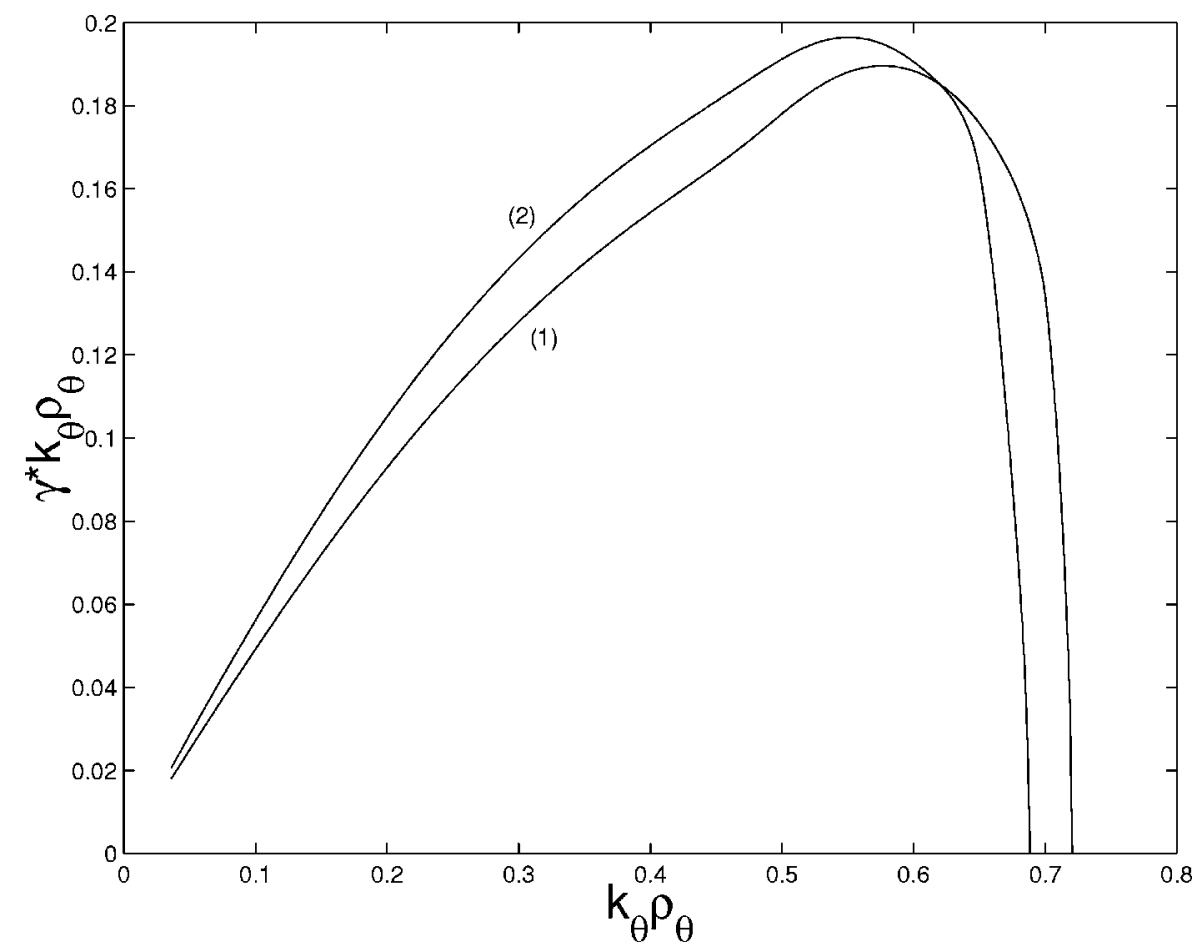

FIG. 5. The dependence of growth rate $\gamma$ (normalized by $\omega_{*}$ ) as a function of $k_{\theta} \rho_{\theta}$ with fixed parameters $\kappa(=1,1.05)$. Curve (1) has $\kappa=1.05$ and curve (2) has $\kappa=1.0$. The other parameters are $\beta=0.004, q=2, \tau=1, s=0.5$, $\varepsilon_{n}=0.5, \eta_{i e}=3$, and $\Delta=0.0$.

such as elongation $\kappa$ and the Shafranov shift $\Delta$ are linked through the magnetic drift frequency $\omega_{D}$, the scale factor $\sqrt{\widetilde{g}_{\theta \theta}}$, and through $k_{\perp}^{2}$ in the eigenvalue differential equation (44). In the limit $\beta \rightarrow 0$ the eigenvalue equation (44) reduces to a local dispersion relation $Q(k, \bar{\omega}, \theta=0)=1 / \tau$ for the electrostatic $\eta_{i}$ mode. The ideal MHD ballooning mode equation can be recovered from Eq. (44) in the limit $|\omega| \gg \omega_{*_{i}}, \omega_{D}$ $(|Q| \ll 1)$. The potential function $V$ then takes the form

$$
V(k, \bar{\omega}, \theta)=\varepsilon_{n} \widetilde{g}_{\theta \theta}\left(1+\eta_{e}+\frac{1}{\tau}\left(1+\eta_{i}\right)\right)+\tau b \bar{\omega}^{2},
$$

and Eq. (44) reduces to the ideal MHD equation

$$
\frac{1}{h_{\theta}} \frac{d}{d \theta}\left(\frac{k_{\perp}^{2}(\theta)}{k_{\theta}^{2}}\right) \frac{1}{h_{\theta}} \frac{d \phi}{d \theta}+\alpha \widetilde{g}_{\theta \theta} \phi+\frac{\omega^{2}}{\omega_{A}^{2}} \frac{k_{\perp}^{2}(\theta)}{k_{\theta}^{2}} \phi=0,
$$

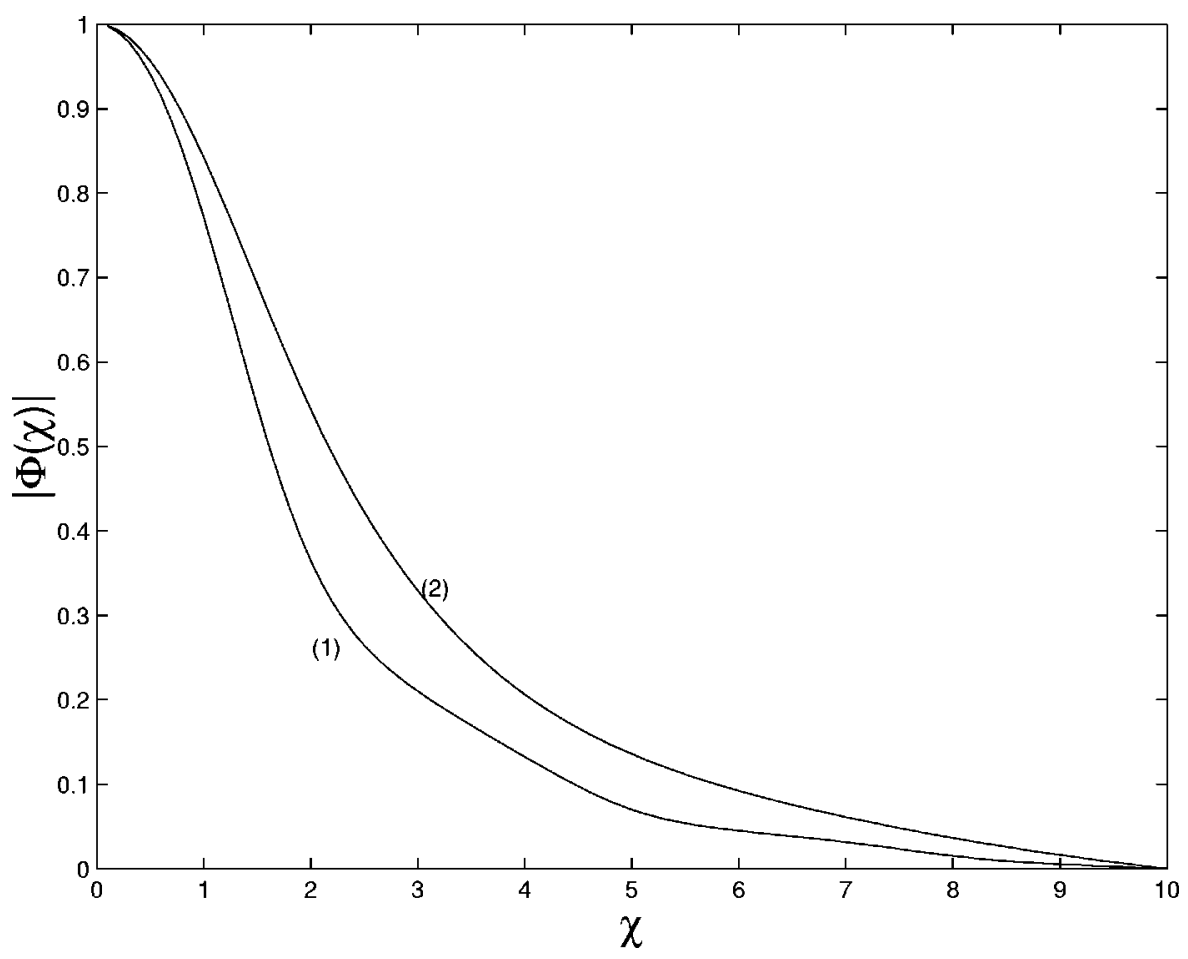

FIG. 6. Eigenfunction $|\Phi|$ as a function of the extended poloidal angle $\chi$. In curves (1) has $\kappa=1$ and curve (2) has $\kappa=1.2$. The other parameters are $\varepsilon=0.2, \beta=0.001, q=2, \tau=1.0, s=0.5$, $k^{2} \rho^{2}=0.1$ and $\eta=3$ and $\Delta=-0.0$. 


$$
\alpha=\frac{\beta q^{2} R}{L_{n}}\left(1+\eta_{e}+\frac{1+\eta_{i}}{\tau}\right) \frac{\tau}{\tau+1},
$$

where $\omega_{A}=1 / q R V_{A}$ is the Alfvén frequency and $\omega_{* e}^{2} / \omega_{A}^{2}$ $=\beta 2 /(1+1 / \tau) q^{2} k_{\theta}^{2} \rho_{s}^{2} / \varepsilon_{n}^{2}$ has been used. Corrections to the ideal MHD-ballooning mode equation (50) arising from perpendicular compressibility were calculated in Ref. 21 using a kinetic model and a two fluid model similar to the one used here (but neglecting the contribution from the diamagnetic heat flow). These compressibility terms lead to instability below the MHD $\beta$ limit for the MHD ballooning mode branch.

\section{SIMULATION RESULTS}

The eigenvalue equation (44) with boundary conditions in toroidal geometry (48) has been solved numerically by a fourth order Runge-Kutta solver combined with a standard shooting technique. The shooting begins at $\theta=0$, where $\Phi^{\prime}(0)=0$ and the complex eigenvalue is iterated until the boundary condition $\Phi \rightarrow 0$ as $\theta \rightarrow \infty$ is met. The equilibrium model considered here demonstrates effects of finite $\beta$ stabilization, Shafranov shift in circular and noncircular geometry. They are the standard $s-\alpha$ model. An isothermal discharge $\left(T_{i}=T_{e}\right)$ with $\eta=\eta_{i}=\eta_{e}$ has been assumed throughout this study. Figure 1 shows a $\beta$ scaling against growth rate $\gamma$ with parameters $\kappa(=1 ., 1.5)$ and Shafranov shift $\Delta(=-0.0$, -0.1 ). Curves (1) and (2) have $\Delta=-0.1$ and $\Delta=-0.0$ respectively with fixed $\kappa=1.5$. Curves (3) and (4) have $\Delta=-0.1$ and $\Delta=-0.0$ respectively with fixed $\kappa=1.0$. The other parameters are $q=2, \tau=1, s=0.5, \eta=2.0, \varepsilon_{n}=0.5, k^{2} \rho^{2}=0.1$. In the elongated case, in curves (1) and (2) with $\kappa=1.5$ the $\beta$ thresholds $(\beta$ critical) shows substantial dependence on Shafranov shift $\Delta$ ). The growth rate is slightly higher without Shafranov shift $(\Delta=-0.0)$ near the electrostatic limit, (i.e., as $\beta$ approaches zero). In circular geometry [curves (3) and (4)] with $\kappa=1$ the $\beta$ thresholds depends strongly on Shafranov shift as predicted in earlier works. We note that the critical value of $\beta$ is improved substantially in the elongated case as compared with circular geometry [curves (1) and (3)]. As $\beta \rightarrow 0$ the electromagnetic ITG mode tends towards the electrostatic limit. Elongation may be stabilizing or destabilizing depending on $\varepsilon_{n}$. However, for a given large $\beta$ with elongation effects, the ITG mode is expected to reduce anomalous ITG-mode transport in toroidal devices. Next we consider the dependence of the mode growth on $\eta=\eta_{i}=\eta_{e}$ and compare in the circular and noncircular cases. Figure 2 shows the growth rates as functions of $\eta=\eta_{i}=\eta_{e}$ with $\kappa=(1.0,1.5)$ and $\Delta=(-0.0,-0.1)$ as parameters. Curves (1) and (3) have $\Delta=-0.1$ and $\Delta=-0.0$, respectively, with fixed $\kappa=1.5$ and fixed $\beta=0.2 \%$. Curves (2) and (4) have $\Delta=-0.1$ and $\Delta=-0.0$, respectively, with fixed $\kappa=1.0$ and fixed $\beta=0.3 \%$. The other parameters are $\varepsilon_{n}=0.5, s=0.5, q=2, \tau=1, k^{2} \rho^{2}$ $=0.1$. In both circular [curves(2) and (4)] and elongated [curves(1) and (3)] cases the ITG mode is stabilizing with dependence on Shafranov shift. We now show the effects of finite $\beta$ on the ITG mode for arbitrary $\kappa, \Delta$, and $\beta$. Figure 3 shows the growth rates as functions of $\eta=\eta_{i}=\eta_{e}$ with $\kappa$ $=(1,1.05)$ and $\Delta=(-0.0,-0.005)$ as parameters. Curves (1) and (2) have $\Delta=-0.005$ and $\Delta=-0.0$, respectively, with fixed $\kappa=1.05$. Curves (3) and (4) have $\Delta=-0.005$ and $\Delta=-0.0$, respectively, with fixed $\kappa=1.0$ (circular case). The other parameters are $\varepsilon_{n}=0.5, \beta=0.4 \%, s=0.5, q=2, \tau=1, k^{2} \rho^{2}=0.1$. Here the lower stability threshold $\eta_{\text {ilow }}$ shows weak dependence on both $\Delta$ and $\kappa$ for fixed $\beta=(0.4 \%$ and slightly stronger dependence on the upper threshold $\left.\eta_{\text {iup }}\right)$. However, there is a strong $\beta$ sensitivity when comparing curves (1-4). In Fig. 4 we show the growth rate $\gamma$ as a function of Shafranov shift $\Delta$ in circular and noncircular geometry. Curves (1) and (2) have $\kappa=1.0$ and $\kappa=1.5$, respectively, with $\eta=2.0$. Curves (3) and (4) have $\kappa=1.0$ and $\kappa=1.5$, respectively, with $\eta$ $=4.0$. The other parameters are $\varepsilon_{n}=0.5, \beta=0.001, s=0.5, q$ $=2, \tau=1, k^{2} \rho^{2}=0.1$. Shafranov shift has in general the weak effect on the eigenfrequency both in the circular and elongated cases. Next we display effects of elongation $\kappa$ on $k_{\theta}$ spectrum. Figure 5 shows mode growth as a function of $k_{\theta} \rho$ with $\kappa$ as parameter. Curves (1) and (2) have $\kappa=1.05$ and $\kappa=1.0$, respectively. For $k_{\theta} \rho=0.55$ we have the maximum growth rate in the circular case $(\kappa=1)$. However, the peak is shifted towards larger $k_{\theta} \rho$ in the elongated case curve (1). In Fig. 6 the modulus of the eigenfunction is shown as a function of the extended poloidal angle $\chi$ for $\kappa(=1,1.2)$. Curves (1) and Curve (2) have $\kappa=1.20$ and $\kappa=1$, respectively. The other parameters are $\varepsilon_{n}=0.2, \beta=0.001, \eta=\eta_{i}=\eta_{e}=3, q=2$, $s=0.5, \tau=1$. The mode tend to be localized in both circular and elongated cases for large $\eta=3$.

\section{SUMMARY}

The $\eta_{i}$ mode with emphasis on the lower $\eta_{\text {ilow }}$ and higher $\eta_{\text {iup }}$ threshold values with elongation $\kappa$, finite $\beta$ effects and Shafranov shift $\Delta$ has been studied by an advanced fluid model. ${ }^{11}$ The derived eigenvalue equation (44), which includes elongation effects $\kappa$ and Shafranov shift $\Delta$ is solved numerically. Boltzmann distributions for the electrons is assumed. Elongation effects enter through Eq. (30), by modifying the magnetic drift frequency $\omega_{D e}$. It is shown that these (combined) effects reduce the second stability threshold significantly whereas a weak dependence on the lower stability regime is shown by these effects. Stabilization is also sensitive to FLR and on $\varepsilon_{n}=2 L_{n} / L_{B}$. The results of the linear studies presented here may be relevant for the nonlinear regime which is emphasized by nonlinear investigations of the $\eta_{i}$ mode turbulence, see for example, Refs. 22 and 23. The $\eta_{i}$ threshold stabilizations studied here may be important in the creation of enhanced confinement regimes. Elongation, Shafranov shift and finite $\beta$ stabilization mechanism may be dominant in situations with aligned transport barriers. In order that these stabilization schemes be relevant in realistic situation other effects such as triangularity, reverse shear, trapping and collisional dissipation may be important. ${ }^{24,25}$

${ }^{1}$ D. D. Hua, Y. Q. Yu, and T. K. Fowler, Phys. Fluids B 4, 3216 (1992).

${ }^{2}$ G. Rewoldt, W. M. Tang, and M. S. Chance, Phys. Fluids 25, 480 (1982).

${ }^{3}$ D. D. Hua, X. Q. Xu, and T. K. Fowler, Phys. Fluids B 4, 10 (1992).

${ }^{4}$ J. Anderson, H. Nordman, and J. Weiland, Phys. Plasmas 8, 180 (2001).

${ }^{5}$ A. Jarmén, P. Andersson, and J. Weiland, Nucl. Fusion 27, 941 (1987).

${ }^{6}$ A. Jarmén, P. Malinov, and H. Nordman, Plasma Phys. Controlled Fusion 40, 2041 (1998).

${ }^{7}$ R. E. Waltz and R. L. Miller, Phys. Plasmas 6, 4265 (1999). 
${ }^{8}$ The JET team and T. T. C. Jones, Phys. Plasmas 4, 1725 (1997).

${ }^{9}$ X.-H. Wang, P. H. Diamond, and M. N. Rosenbluth, Phys. Fluids B 4, 2402 (1992).

${ }^{10}$ C. Z. Cheng, Phys. Fluids 25, 1020 (1982).

${ }^{11} \mathrm{~J}$. Weiland, Collective Modes in Inhomogeneous Plasmas, Kinetic and Advanced Fluid Theory (IOP Bristol, 2000), pp. 123-125.

${ }^{12}$ P. Andersson and J. Weiland, Phys. Fluids 31, 359 (1988).

${ }^{13}$ A. Jarmén, P. Andersson, and J. Weiland, Nucl. Fusion 25, 941 (1987).

${ }^{14}$ G. Bateman, A. H. Kritz, J. E. Kinsey, A. J. Redd, and J. Weiland, Phys. Plasmas 5, 1793 (1998)

${ }^{15}$ A. M. Dimits, G. Bateman, M. A. Beer, B. I. Cohen et al., Phys. Plasmas 7, 969 (2000)

${ }^{16}$ R. L. Miller, M. S. Chou, J. M. Greene et al., Phys. Plasmas 5, 973 (1998).
${ }^{17}$ W. D. D'haeseler, W. N. G. Hitchon, J. D. Callen, Flux Coordinates and Magnetic Field Structure (Springer, Berlin, 1991).

${ }^{18}$ M. Nakahara, Geometry, Topology and Physics (IOP, Bristol, 1990).

${ }^{19}$ H. Nordman, B. Jhowry, and J. Weiland, Phys. Fluids B 5, 3465 (1993).

${ }^{20}$ J. W. Connor, R. J. Hastie, and J. B. Taylor, Phys. Rev. Lett. 40, 396 (1978).

${ }^{21}$ R. R. Dominguez and R. W. Moore, Nucl. Fusion 26, 85 (1986).

${ }^{22}$ S. E. Parker, W. W. Lee, and R. A. Santoro, Phys. Rev. Lett. 71, 2042 (1993).

${ }^{23}$ R. E. Waltz, G. D. Kerbel, J. Milovich, and G. W. Hammett, Phys. Plasmas 2, 2048 (1995).

${ }^{24}$ W. M. Tang, G. Rewoldt, and M. S. Chance, Nucl. Fusion 25, 151 (1985).

${ }^{25}$ J. Weiland and A. Hirose, Nucl. Fusion 32, 151 (1992). 